\title{
ON THE AUTOMORPHISM GROUP OF A FINITE MODULAR $p$-GROUP
}

\author{
RICHARD M. DAVITT AND ALBERT D. OTTO
}

\begin{abstract}
In this paper it is shown that if $G$ is a finite nonAbelian modular $p$-group $(p>2)$, then the order of $G$ divides the order of the automorphism group of $G$.
\end{abstract}

In recent years there have been a number of papers exploring the relationships between the order of a finite $p$-group and the order of its automorphism group. In particular many of these have shown that for a certain class of finite $p$-groups, the order of the group divides the order of the automorphism group ([1], [2], [3], [4], [8]). We recall that a group $G$ is modular if the lattice of subgroups of $G$ is modular. It is the purpose of this paper to show that if $G$ is a non-Abelian modular $p$-group $(p>2)$, then the order of $G$ divides the order of the automorphism group of $G$.

The following notation is used: $G$ is a finite $p$-group where $p$ is an odd prime; $A(G)$ is the group of automorphisms of $G ; A_{c}(G)$ and $\operatorname{Inn}(G)$ are the normal subgroups of $A(G)$ of central automorphisms and inner automorphisms, respectively; $G_{n}$ is the $n$th member of the lower central series of $G$ and so $G_{2}$ is the derived group; $Z(G)$ and $Z_{2}(G)$ are the center and second center of $G$, respectively (or $Z$ and $Z_{2}$ if no ambiguity is possible); $\exp G$ is the exponent of $G ;|G|$ is the order of the group $G$; for $x$ and $y$ in $G, o(x)$ is the order of the element $x,(x, y)$ is the commutator $x^{-1} y^{-1} x y$, and $\langle x\rangle$ is the cyclic subgroup generated by $x ; \Omega_{n}(G)=$ $\left\{x \in G: o(x) \leqq p^{n}\right\} ;$ and $\mho_{n}(G)=\left\{x^{p^{n}}: x\right.$ in $\left.G\right\}$. In addition, $H \leqq G$ means $H$ is a subgroup of $G$ and $\oplus$ is used to denote the direct sum of subgroups. Finally, for groups $A$ and $B, \operatorname{Hom}(A, B)$ is the set of all homomorphisms from $A$ into $B$.

There are certain results which we often need and shall use throughout the paper without further reference. Their proofs may be found in [5]. If $a, b$, and $c$ are elements of a group $G$, then $(a, b c)=(a, c)(a, b)^{c}$ and $(a b, c)=(a, c)^{b}(b, c)$. Also $\left|\operatorname{Hom}\left(\oplus A_{i}, \oplus B_{j}\right)\right|=\prod_{i, j}\left|\operatorname{Hom}\left(A_{i}, B_{j}\right)\right|$ where $A_{i}$ and $B_{j}$ are Abelian $p$-groups. In addition, $\operatorname{Hom}\left(C\left(p^{\alpha}\right), C\left(p^{\beta}\right)\right)$ is isomorphic to $C\left(p^{\min (\alpha, \beta)}\right)$ where $C(n)$ is the cyclic group of order $n$. Finally,

Presented to the Society, January 18, 1972; received by the editors October 11, 1971 . AMS 1970 subject classifications. Primary 20D15, 20D45; Secondary 20F55.

Key words and phrases. Finite $p$-groups, regular, modular, inner automorphisms, central automorphisms. 
it is assumed that the definition of a regular p-group and the basic properties of such groups are known.

We now state our theorem.

THEOREM. If $G$ is a finite non-Abelian modular p-group $(p>2)$, then $|G|$ divides $|A(G)|$.

We may assume that the nilpotency class of $G$ is greater than $2[4]$ and also that $G / Z$ is not metacyclic [3]. Furthermore, we may assume $G$ is purely non-Abelian $[8]$ and consequently $\left|A_{c}(G)\right|=|\operatorname{Hom}(G, Z)|[1]$ which is then a power of $p$.

For purposes of notation we shall let $R$ be the normal subgroup $\operatorname{Inn}(G) A_{c}(G)$ of $A(G)$. We note that $R$ is a $p$-group and our goal is to show that $|R| \geqq|G|$.

We begin by examining in greater detail the structure of $G$. By [6] and [7] or [9] we know that $G$ contains a normal Abelian subgroup $A$ and an element $b$ such that $G \mid A=\langle b A\rangle$ and for some fixed nonnegative integer $\alpha,(a, b)=a^{p^{\alpha}}$ for each $a$ in $A$. Since $G / A$ is Abelian, $G_{2}$ is contained in $A$. Also $\alpha>0$. For if $\alpha=0,(a, b)=a$ for each $a$ in $A$ and so $G_{2}=A$ which is a contradiction since $G / A$ is cyclic. Since $(a, b)=a^{p^{\alpha}}$ for each $a$ in $A$, $\mho_{\alpha}(A) \leqq G_{2}$. We next note that $\left(a, b^{i}\right)=a^{\left(1+p^{\alpha}\right)^{i-1}}$ is in $\mho_{\alpha}(A)$ for each $a$ in $A$ and integer $i \geqq 0$. Thus by using commutator identities we have that each commutator is of the form $a^{p^{\alpha}}$ for some $a$ in $A$. Thus $\mho_{\alpha}(A)=G_{2}$. In a similar fashion we see that $\mho_{2 \alpha}(A)=G_{3}$. We can now prove the following important and useful lemma.

LemMA 1. G is regular.

Suppose $x$ and $y$ are in $G$ and $H=\langle x, y\rangle$. For convenience we may assume $H$ is not Abelian. Then $(x y)^{p}=x^{p} y^{p} c^{p} d$ where $c$ is in $H_{2}$ and $d$ is in $H_{p}$. Since $G$ is modular, $H$ is also modular. Thus we have $H_{p} \leqq H_{3}=\mho_{\alpha_{1}}\left(H_{2}\right) \leqq$ $\mho_{1}\left(H_{2}\right)$, where $\alpha_{1}$ is the " $\alpha$ " for $H$. Thus $\bar{d}=\bar{d}^{p}$ where $\bar{d}$ is in $H_{2}$. Since $H_{2} \leqq G_{2}$ which is Abelian $c^{p} d=(c \bar{d})^{p}$ with $c \bar{d}$ in $H_{2}$. So $(x y)^{p}=x^{p} y^{p} z^{p}$ with $z$ in $H_{2}$ and $G$ is regular.

For purposes of notation we let $p^{k}=|G| A \mid$ and $p^{m}=\exp G_{2}$. Since $G$ is not Abelian, $G_{2}=\mho_{\alpha}(A)>E$ and so $\exp A=p^{m+\alpha}$. Also since for each $a$ in $A, e=(a, b)^{p^{m}}=\left(a, b^{p^{m}}\right)=\left(a^{p^{m}}, b\right)$, we see that $a^{p^{m}}$ and $b^{p^{m}}$ are in $Z$ and hence that $\mho_{m}(G) \leqq Z$. Consequently, $m>\alpha$. For if $m \leqq \alpha$, then $G_{2}=\mho_{\alpha}(A) \leqq \mho_{\alpha}(G) \leqq \mho_{m}(G) \leqq Z$, which means $G$ has nilpotency class 2 . We also observe that since for each $a$ in $A,(a, b)=a^{p^{\alpha}}$, we have $a^{p^{\alpha}}=e$ if and only if $a$ is in $Z$. Thus $\Omega_{\alpha}(A)=A \cap Z$.

In order to find a suitable lower bound on $|R|$, we begin by determining $Z$ and $Z_{2}$ and consequently their orders. 
First of all we recall that $b^{p^{m}}$ is in $Z$ and thus $\left\langle b^{p^{m}}\right\rangle(A \cap Z) \leqq Z$. Conversely suppose $x=b^{i} a$ with $a$ in $A$ is in $Z$. Then $e=(x, b)=\left(b^{i} a, b\right)=$ $(a, b)=a^{p^{\alpha}}$ and so $a$ is in $\Omega_{\alpha}(A)$. For $\bar{a}$ in $A, e=\left(b^{i} a, \bar{a}\right)=\left(b^{i}, \bar{a}\right)=(b, \bar{a})^{i}$ and so $p^{m} \mid i$. Thus $Z=\left\langle b^{p^{m}}\right\rangle(A \cap Z)=\left\langle b^{p^{m}}\right\rangle \Omega_{\alpha}(A)$. We note that, since $o(b A)=p^{k}, o(b(A \cap Z))=p^{k}$. Furthermore $o(b Z)=p^{m}$. For if $o(b Z) \leqq p^{m-1}$, then, by the regularity of $G$, each commutator in $G$ has order $\leqq p^{m-1}$ and so $\exp G_{2} \leqq p^{m-1}$. Thus in addition we have $k \geqq m$ and $|Z|=p^{k-m}\left|\Omega_{\alpha}(A)\right|$.

Next we investigate $Z_{2}$ and find its order. First of all it is easily seen that $Z_{2}$ can be characterized as the set of all $x$ in $G$ such that $(x, b) \in Z$ and $(x, a) \in Z$ for all $a$ in $A$. For $a$ in $\Omega_{2 \alpha}(A),(a, b)=a^{p^{\alpha}}$ is in $\Omega_{\alpha}(A) \leqq Z$. Thus, $\Omega_{2 \alpha}(A) \leqq Z_{2}$. Also for $a$ in $A,\left(a, b^{p^{m-\alpha}}\right)$ is in $\left\langle a^{p^{m}}\right\rangle \leqq Z$. Thus $\left\langle b^{p^{m-\alpha}}\right\rangle \Omega_{2 \alpha}(A) \leqq Z_{2}$. Conversely suppose $x=b^{i} a$ is in $Z_{2}$ with $a$ in $A$. Then $(x, b)=\left(b^{i} a, b\right)=(a, b)=a^{p^{\alpha}}$ is in $Z \cap A=\Omega_{\alpha}(A)$ and so $a$ is in $\Omega_{2 \alpha}(A)$. So now $b^{i}$ is in $Z_{2}$ and so for $\bar{a}$ in $A,\left(b^{i}, \bar{a}\right)$ is in $A \cap Z=\Omega_{\alpha}(A)$. Thus $e=\left(b^{i}, \bar{a}\right)^{p^{\alpha}}=\left(b^{i p^{\alpha}}, \bar{a}\right)$. So $b^{i p^{\alpha}}$ is in $Z$ and $p^{m} \mid i p^{\alpha}$. Hence $p^{m-\alpha} \mid i$. So now $Z_{2}=\left\langle b^{p^{m-\alpha}}\right\rangle \Omega_{2 \alpha}(A)$. Since $o(b A)=o\left(b \Omega_{\alpha}(A)\right)=p^{k}$, we have $o\left(b \Omega_{2 \alpha}(A)\right)=p^{k}$. Thus $\left|Z_{2}\right|=p^{k-m+\alpha}\left|\Omega_{2 \alpha}(A)\right|$.

We can now give our first estimate on $|R|$.

$$
\begin{aligned}
|R| & =\left|\operatorname{Inn}(G) A_{c}(G)\right|=|\operatorname{Inn}(G)|\left|A_{c}(G)\right| /\left|Z_{2} / Z\right| \\
& =\left([|G| /|Z|]\left|A_{c}(G)\right|\right) /\left(\left|Z_{2}\right| /|Z|\right)=p^{k}|A|\left|A_{c}(G)\right| / p^{k-m+\alpha}\left|\Omega_{2 \alpha}(A)\right| \\
& =p^{m-\alpha}\left|A_{c}(G)\right|\left(|A| /\left|\Omega_{2 \alpha}(A)\right|\right)=p^{m-\alpha}\left|A_{c}(G)\right|\left|\mho_{2 \alpha}(A)\right| .
\end{aligned}
$$

We now turn our attention to finding a suitable lower bound for $\left|A_{c}(G)\right|$. To do this we look at $|\operatorname{Hom}(G, Z)|$ which is the same as $\left|\operatorname{Hom}\left(G / G_{2}, Z\right)\right|$. For this purpose we shall examine $A$ as well as $G / G_{2}$ and $Z$.

Since $\exp A=p^{m+\alpha}, A$ may be written in the form $\left\langle a_{1}\right\rangle S$ where $o\left(a_{1}\right)=$ $p^{m+\alpha}$ and $S$ is a subgroup. We observe that $\exp S>p^{\alpha}$. For if not, then $S \leqq \Omega_{\alpha}(A)=A \cap Z \leqq Z$ and so $G / Z$ would be metacyclic with generators $a_{1} Z$ and $b Z$. Consequently $S=\left\langle a_{2}\right\rangle \oplus T$ where $T$ is a subgroup of order $p^{r}$, $r \geqq 0$, and $o\left(a_{2}\right)=p^{m_{2}+\alpha}$ with $\left.m_{2}\right\rangle 0$. Hence, $A=\left\langle a_{1}\right\rangle \oplus\left\langle a_{2}\right\rangle \oplus T$.

We next turn our attention to $G / G_{2}$. Let us suppose $G / G_{2}=\left\langle\bar{b}_{1}\right\rangle \oplus \cdots \oplus$ $\left\langle\bar{b}_{v}\right\rangle$ where $\bar{b}_{i}=b_{i} G_{2}$ with $b_{i}$ in $G$ and $o\left(\bar{b}_{i}\right)=p^{k_{i}}$ with $k_{1} \geqq k_{2} \geqq \cdots \geqq k_{v}$. First of all we observe that $v \geqq 3$. For otherwise $G$ can be generated by the two elements $b$ and $a_{1}$ and hence would be metacyclic. We now prove a sequence of three lemmas each of which will establish an important relationship to be used in estimating $\left|A_{c}(G)\right|$.

LEMMA 2. $o\left(b G_{2}\right)=p^{k_{1}}$ and so $k+\alpha \geqq k_{1}$.

Since $\exp G / G_{2}=p^{k_{1}}, o\left(b G_{2}\right) \leqq p^{k_{1}}$. Furthermore, there is $g=b^{i} a$ with 
$0 \leqq i<p^{k}$ and $a$ in $A$ such that $o\left(g G_{2}\right)=p^{k_{1}}$. Because $G_{2} \leqq A, p^{k}=o(b A) \leqq$ $o\left(b G_{2}\right) \leqq p^{k_{1}}$ and so $k_{1} \geqq k>\alpha$. So $e \neq\left(g G_{2}\right)^{p^{\alpha}}=\left(b^{i} a G_{2}\right)^{p^{\alpha}}=\left(b^{i p^{\alpha}} a^{p^{\alpha}}\right) G_{2}=$ $b^{i p^{\alpha}} G_{2}=\left(b^{i} G_{2}\right)^{p^{\alpha}}$ since $a^{p^{\alpha}} \in \mho_{\alpha}(A)=G_{2}$. From $\left(b^{i} G_{2}\right)^{p^{\alpha}}=\left(g G_{2}\right)^{p^{\alpha}} \neq e$, we can conclude that $o\left(b G_{2}\right) \geqq p^{k_{1}}$ and thus $o\left(b G_{2}\right)=p^{k_{1}}$. Since $o\left(b \Omega_{\alpha}(A)\right)=p^{k}$, $o(b) \leqq p^{k+\alpha}$. Now with $o\left(b G_{2}\right)=p^{k_{1}}$, we see that $k_{1} \leqq k+\alpha$.

Thus we may now assume without loss of generality that $b=b_{1}$. Furthermore we observe that $k_{1} \geqq k$.

LEMMA 3. $\quad p^{k_{1}} \geqq \exp Z$.

For the purpose of notation in this lemma let $\exp Z=p^{l}$. We recall that $\Omega_{\alpha}(A) \leqq Z$ and thus $l \geqq \alpha$. If $\alpha=l$, then since $\alpha<k \leqq k_{1}$, we have that $k_{1} \geqq l$. Thus we may assume that $l>\alpha$. We now recall that $Z=\left\langle b^{p^{m}}\right\rangle \Omega_{\alpha}(A)$ and since $\exp Z>\exp \Omega_{\alpha}(A)$, we have that $o\left(b^{p^{m}}\right)=p^{l}$ and so $o(b)=p^{m+l}$. Since $\exp G_{2}=p^{m}$ and $o\left(b G_{2}\right)=p^{k_{1}}$, we have $b^{p^{m+k_{1}}}=e$. Hence $m+k_{1} \geqq m+l$ and so $k_{1} \geqq l$.

LEMMA 4. $k_{2}=\alpha$.

Let $b_{2}=b_{1}^{i} a$ where $b_{1}=b, 0 \leqq i<p^{k}$, and $a$ is in $A$. Then

$$
\left(b_{2} G_{2}\right)^{p^{\alpha}}=\left(b_{1}^{i} a G_{2}\right)^{p^{\alpha}}=\left(b_{1}^{i p^{\alpha}} a^{p^{\alpha}}\right) G_{2}=b_{1}^{i p^{\alpha}} G_{2}=\left(b_{1} G_{2}\right)^{i p^{\alpha}}
$$

since $a^{p^{\alpha}}$ is in $\mho_{\alpha}(A)=G_{2}$. Because of the direct sum in $G / G_{2}$, we have $\left(b_{2} G_{2}\right)^{p \alpha}=e$ and so $\alpha \geqq k_{2}$. Now by way of contradiction suppose $k_{2}<\alpha$. Since $\bar{b}_{1}, \cdots, \bar{b}_{v}$ generate $G / G_{2}, b_{1}, b_{2}, \cdots, b_{v}$ generate $G$ and hence $G_{2}$ is the normal closure of $\left\{\left(b_{i}, b_{j}\right): 1 \leqq i<j \leqq v\right\}$. For $i \geqq 2$, we have $o\left(b_{i} G_{2}\right)=$ $p^{k_{i}} \leqq p^{k_{2}} \leqq p^{\alpha-1}$ and thus $b_{i}^{p^{\alpha-1}}$ is in $G_{2} \leqq A$. So when $i \geqq 2\left(b_{i}^{p^{\alpha-1}}\right)^{p^{m}}=e$ since $\exp G_{2}=p^{m}$. Consequently, for $i \geqq 2,\left(b_{i}^{p^{\alpha-1}}\right)^{p^{m-\alpha}}=b_{i}^{p^{m-1}}$ is in $\Omega_{\alpha}(A) \leqq$ $Z$ and hence $o\left(b_{i} Z\right) \leqq p^{m-1}$. So $o\left(b_{i}, b_{j}\right) \leqq p^{m-1}$ when $1 \leqq i<j \leqq v$. This means $\exp G_{2} \leqq p^{m-1}$ since $G$ is regular. This is a contradiction. Thus we now have $k_{2}=\alpha$.

For convenience we now let $H=\left\langle\bar{b}_{3}\right\rangle \oplus \cdots \oplus\left\langle\bar{b}_{v}\right\rangle$ and let $|H|=p^{s}$. We note that $s>0$ since $v \geqq 3$. Also we can now calculate $\left|G / G_{2}\right|$ in two ways. Since $G / G_{2}=\left\langle\bar{b}_{1}\right\rangle \oplus\left\langle\bar{b}_{2}\right\rangle \oplus H$, we have

$$
\left|G / G_{2}\right|=p^{k_{1}+\alpha+s} .
$$

Furthermore, $\left|G / G_{2}\right|=\left|G / \mho_{\alpha}(A)\right|=|G| A|| A / \mho_{\alpha}(A) \mid$ and thus we have

$$
\left|G / G_{2}\right|=p^{k}\left|\Omega_{\alpha}(A)\right| .
$$

Taking a careful look at $\left|A_{c}(G)\right|$, we have $\left|A_{c}(G)\right|=\left|\operatorname{Hom}\left(G / G_{2}, Z\right)\right|=\left|\operatorname{Hom}\left(\left\langle\bar{b}_{1}\right\rangle, Z\right)\right|\left|\operatorname{Hom}\left(\left\langle\bar{b}_{2}\right\rangle, Z\right)\right||\operatorname{Hom}(H, Z)|$. 
By Lemma 3, $\left|\operatorname{Hom}\left(\left\langle\bar{b}_{1}\right\rangle, Z\right)\right|=|Z|$. By Lemma 4, $\left|\operatorname{Hom}\left(\left\langle\bar{b}_{2}\right\rangle, Z\right)\right|=$ $\left|\Omega_{\alpha}(Z)\right| \geqq\left|\Omega_{\alpha}(A)\right|$. Since $\exp Z \geqq p^{\alpha}=p^{k_{2}} \geqq p^{k_{3}} \geqq \cdots \geqq p^{k_{\mathfrak{v}}}$, we have

$$
|\operatorname{Hom}(H, Z)| \geqq p^{s} \text {. }
$$

Hence we now see that $\left|A_{c}(G)\right| \geqq|Z|\left|\Omega_{\alpha}(A)\right| p^{s} \geqq p^{k-m}\left|\Omega_{\alpha}(A)\right|^{2} p^{s}$.

This combined with our earlier calculation of $|R|$ yields

$$
\begin{aligned}
|R| & \geqq p^{m-\alpha}\left|\mho_{2 \alpha}(A)\right| p^{k-m}\left|\Omega_{\alpha}(A)\right|^{2} p^{s} \\
& \geqq p^{k-\alpha+s}\left|\mho_{2 \alpha}(A)\right|\left|\Omega_{\alpha}(A)\right|^{2} \\
& \geqq p^{k-\alpha+s}|A| .
\end{aligned}
$$

First of all let us suppose that $r \geqq \alpha$ where we recall that $o(T)=p^{r}$. If $\exp T \geqq p^{\alpha}$, then $\left|\Omega_{\alpha}(A)\right| \geqq p^{\alpha+\alpha+\alpha}=p^{3 \alpha}$. If $\exp T<p^{\alpha}$, then $T \leqq \Omega_{\alpha}(A)$ and so $\left|\Omega_{\alpha}(A)\right|=p^{\alpha+\alpha}|T|=p^{\alpha+\alpha+r} \geqq p^{3 \alpha}$. In either case $\left|\Omega_{\alpha}(A)\right| \geqq p^{3 \alpha}$. Then using (2) we see that $\left|G / G_{2}\right| \geqq p^{k+3 \alpha}$. Consequently, we have $k_{1}+\alpha+s \geqq k+3 \alpha$ from (1). Then Lemma 2 implies that $k+\alpha+\alpha+s \geqq k+3 \alpha$ or that $s \geqq \alpha$. Since $|G|=p^{k}|A|$ we now have $|R| \geqq|G|$ by (4). Thus we may assume $r<\alpha$ and hence just as important we now have $T \leqq \Omega_{\alpha}(A)$.

We recall that $A=\left\langle a_{1}\right\rangle \oplus\left\langle a_{2}\right\rangle \oplus T$ where $o\left(a_{1}\right)=p^{m+\alpha}$ and $o\left(a_{2}\right)=p^{m_{2}+\alpha}$ with $m_{2}>0$. Thus $\Omega_{\alpha}(A)=\left\langle a_{1}^{p^{m}}\right\rangle \oplus\left\langle a_{2}^{p^{m}}\right\rangle \oplus T$. Since $o\left(b \Omega_{\alpha}(A)\right)=p^{k}, b^{p^{k}}=$ $\left(a_{1}^{p^{m}}\right)^{w_{1}}\left(a_{2}^{p^{m}}\right)^{w_{2}} a$ where $w_{1}, w_{2} \geqq 0$ and $a$ is in $T$. Furthermore $\left|\Omega_{\alpha}(A)\right|=$ $p^{2 \alpha+r}$. We now divide the proof into two major cases.

Case (i). $m_{2} \geqq \alpha$. Then $b^{p^{k+r}}=a_{1}^{w_{1} p^{m+r}} a_{2}^{w_{2} p^{m_{2}+r}}$ since $\exp T \leqq p^{r}$. Since $m, m_{2} \geqq \alpha$, we have $b^{p^{k+r}}$ is in $\mho_{\alpha}(A)=G_{2}$. Thus $k+r \geqq k_{1}$ because $o\left(b G_{2}\right)=$ $p^{k_{1}}$. Hence, by (1) and (2), $k_{1}+\alpha+s=k+2 \alpha+r$ and so $k+r+\alpha+s \geqq$ $k+2 \alpha+r$ which means $s \geqq \alpha$. Again by (4) we have $|R| \geqq|G|$.

Case (ii). $m_{2}<\alpha$. To finish this case we consider two possibilities.

Subcase (a). $\alpha-m_{2} \leqq r$. Let $r=\alpha-m_{2}+\beta$ where $\beta \geqq 0$. Then $b^{p^{k+r}}=$ $a_{1}^{w_{1} p^{m+\alpha-m_{2}+\beta}} a_{2}^{w_{2} p^{\alpha+\beta}}$ since $a^{p^{r}}=e$. Because $\beta \geqq 0$ and $\alpha>m_{2}, b^{p^{k+r}}$ is in $\mho_{\alpha}(A)=G_{2}$ and as in Case (i), $k+r \geqq k_{1}$ and again as in Case (i), $|R| \geqq|G|$.

Subcase (b). $\quad \alpha-m_{2}>r$. Then $b^{p^{k_{+} \alpha-m_{2}}}=a_{1}^{w_{1} p^{m+\alpha-m_{2}}} a_{2}^{w_{2} p^{\alpha}}$ where $a^{p^{\alpha-m}}=e$ since $\alpha-m_{2}>r$. Again we have $b^{p^{k+x-m_{2}}}$ is in $\mho_{\alpha}(A)=G_{2}$ and so $k+\alpha-m_{2} \geqq$ $k_{1}$. From (1) and (2) we see that $k_{1}+\alpha+s=k+2 \alpha+r$ and thus $k+\alpha-$ $m_{2}+\alpha+s \geqq k+2 \alpha+r$. So now $s \geqq m_{2}+r$. Since $m_{2}<\alpha$ we have that $\mho_{2 \alpha}(A)=\left\langle a_{1}^{p^{2 \alpha}}\right\rangle$ and so $\left|\mho_{2 \alpha}(A)\right|=p^{m-\alpha}$. Thus (3) yields

$$
|R| \geqq p^{k-\alpha+s} p^{m-x}\left(p^{2 \alpha+r}\right)^{2} \geqq p^{k+m+2 \alpha+2 r+s} \geqq p^{k+m+2 \alpha+3 r+m_{2}} .
$$

At the same time

$$
|G|=p^{k}|A|=p^{k} p^{m+x} p^{m_{2}+x} p^{r}=p^{k+m+2 x+m_{2}+r} .
$$

Hence $|R| \geqq|G|$ and the proof is now complete. 


\section{REFERENCES}

1. J. E. Adney and T. Yen, Automorphisms of a p-group, Illinois J. Math. 9 (1965), 137-143. MR 30 \#2072.

2. R. M. Davitt, The automorphism group of finite p-Abelian p-groups, Illinois J. Math. 16 (1972), 76-85.

3. R. M. Davitt and A. D. Otto, On the automorphism group of a finite p-group with the central quotient metacyclic, Proc. Amer. Math. Soc. 30 (1971), 467-472.

4. R. Faudree, A note on the automorphism group of a p-group, Proc. Amer. Math. Soc. 19 (1968), 1379-1382. MR 40 \#1476.

5. B. Huppert, Endliche Gruppen. I, Die Grundlehren der math. Wissenschaften, Band 134, Springer-Verlag, Berlin and New York, 1967. MR 37 \#302.

6. K. Iwasawa, Über die endlichen und die Verbände ihrer Untergruppen, J. Fac. Sci. Imp. Univ. Tokyo Sect. I 4 (1941), 171-199. MR 3, 193.

7. F. Napolitani, Sui p-gruppi modulari finiti, Rend. Sem. Mat. Univ. Padova 39 (1967), 296-303. MR 36 \#6509.

8. A. D. Otto, Central automorphisms of a finite p-group, Trans. Amer. Math. Soc. 125 (1966), 280-287. MR 34 \#4362.

9. G. Seitz, On the structure of modular p-groups, unpublished paper.

Department of Mathematics, University of Louisville, Louisville, Kentucky 40208

Department of Mathematics, Illinois State University, Normal, Illinois 61761 